\title{
Éducation au développement durable, un territoire révélateur
}

Michel Floro

\section{(2) OpenEdition \\ Journals}

Édition électronique

URL : http://journals.openedition.org/ere/833

DOI : $10.4000 /$ ere.833

ISSN : 2561-2271

Éditeur

Centr'ERE

Référence électronique

Michel Floro, «Éducation au développement durable, un territoire révélateur », Éducation relative à

l'environnement [En ligne], Volume 11 | 2014, mis en ligne le 20 décembre 2013, consulté le 21 février 2020. URL : http://journals.openedition.org/ere/833 ; DOI : 10.4000/ere.833 


\title{
Éducation au développement durable, un territoire révélateur
}

\author{
Michel Floro
}

Cet article interroge le niveau de conscience que les enseignants ont du développement durable (DD), abordé sous l'angle des questions socialement vives (QSV) (Legardez, et Simonneaux 2006), questions vives dans la cité, sans cesse en débat et qui, en éducation, présentent le double risque d'enseigner et d'apprendre. Ce travail s'inscrit dans le champ de la professionnalité enseignante confrontée à une injonction d'éducation au développement durable (EDD) lancée en 2008 par le ministère français de l'Éducation nationale. Il cherche à comprendre comment les enseignants se positionnent, en tant que professionnels, mais aussi en tant que citoyens, sujets et acteurs sociaux, face à cette question épineuse : comment éduquer au développement durable ? Notre travail de recherche a pour but de rendre intelligibles les prises de conscience du phénomène à partir d'une approche territorialisée de la question. Bien que la conscience soit une propriété de l'esprit, son contenu est influencé par la société, l'environnement et en particulier par la façon d'investir son espace de vie. Nous cherchons à repérer les éléments d'un processus interactif complexe, entre l'espace physique, son organisation territoriale (sociale, politique, économique), l'individu (humain affectif, historique) et le collectif. Cette recherche a été conduite en France et en Italie entre 2009 et 2011, dans le cadre de l'Agence Nationale de la Recherche ${ }^{1}$, pour repérer les appuis et les obstacles à l'éducation au développement durable, auprès de 135 enseignants du primaire et du secondaire.

\section{Contexte de travail et problématique : une commande institutionnelle et des incidences sur les pratiques éducatives}

2 Pour l'institution, l'éducation au développement durable vise, depuis 2008, un objectif de solidarité internationale. Elle présente une dimension pérenne dans la formation des jeunes et prescrit l'action par la médiation de projets. Le groupe de travail 
interministériel sur l'éducation au développement durable, présidé par Jacques Brégeon, a engagé dans son rapport l'institution éducative française à passer de l'état d'expérimentation à celui de la généralisation de la transmission des savoirs liés au développement durable. En 2009, la démarche 3D ${ }^{2}$ apparaît comme un outil d'action privilégié pour les établissements. Et l'EDD influence de fait les processus d'éducation. Le développement durable (DD), appuyé sur des savoirs non encore scolarisés, agit sur les modalités de transmission et influe sur les pratiques professionnelles. Il opère des transformations de forme scolaire (Audigier, 2008 ; Vincent, 2008) ou de rapport aux savoirs (Legardez, 2006) qui plonge l'éducation dans une quête de sens. L'enseignant devient un régulateur de débat au lieu d'être celui qui sait (Legardez et Simonneaux, 2006). Il éclaire des problématiques sociales complexes dont les enjeux dépassent le temps, l'espace vécu et à vivre et dont l'(ir)réalité touche à la fois les élèves et l'enseignant. Avant de parler d'éducation, le problème pour nous, est de repérer les caractéristiques que les enseignants attribuent au développement durable et d'identifier le débat social où ils le situent. Quelles sont leurs connaissances propres, acquises dans leurs pratiques quotidiennes et sous-jacentes aux processus d'éducation? Les problématiques de DD remettent en cause, en permanence, les savoirs qu'elles interrogent dans divers champs disciplinaires. Mais les foisonnements de points de vue qui en émergent insufflent de l'innovation dans le système et suscitent une variété d'attitudes professionnelles. Pour Legardez et Simonneaux (2011), l'EDD, en tant que question vive, se réfère en partie au domaine de la P.N.S ${ }^{3}$, une science sociale qui place les problématiques humaines au centre de ses préoccupations, se projette dans l'avenir et n'hésite pas à se confronter à l'incertitude de prises de décision urgentes en termes d'enjeux qui engagent le futur de l'humanité. Le DD demande donc de déconstruire les modèles classiques d'enseignement, fondés sur la transmission de vérités normées, sans controverse, et de penser sa double articulation d'une part, avec les processus éducatifs et d'autre part, avec le projet dans lequel la société veut l'engager.

\section{Un double cadre d'approche : questions socialement vives et territoire}

3 Les questions relatives au développement durable ont quelque chose de vital. La nature du champ d'interrogations qu'elles représentent a, de ce fait, toutes les caractéristiques d'une question vive: elles suscitent des débats, elles sont prégnantes dans l'environnement social et posent des problèmes aux enseignants pour les aborder (Legardez et Simonneaux, 2006). Le développement durable fait partie des questions adressées à la communauté humaine et c'est bien parce que ses interrogations vont audelà des problèmes didactiques ou pédagogiques que nous questionnons la façon dont les enseignants le pensent, dans et hors de l'école. Les enjeux sociétaux réels qu'ils affrontent en tant que citoyens pèsent inévitablement dans leur pratique. La nature même des questions rend incontournable leur engagement dans un processus éducatif renouvelé et devient de ce fait un préalable à ce type d'éducation. L'injonction éducative n'engage pas seulement les enseignants dans leur métier, mais aussi dans leur vie et réciproquement, leur engagement dans la vie transforme les processus éducatifs qu'ils mettent en œuvre. Cette injonction, hors de toute spécialité disciplinaire, vient interroger la citoyenneté enseignante dans le cadre même de son métier. 
D'autre part, le contexte territorial participe à la compréhension du problème. Or un ensemble de travaux de recherche récents montrent les effets de territoire sur les processus éducatifs scolaires (Grelet, 2006, Champolion, 2008). Le territoire est pensé comme un espace investi, construit, reconstruit par les acteurs dans la perspective d'une meilleure gouvernance, qui permet de rendre lisibles les stratégies d'adaptation. Un territoire présente une dimension cognitive dans le sens où chaque individu est à la fois marqué par lui selon un processus de territorialisation des esprits (Bozonet, 1992), mais il est aussi le produit d'une appropriation par les acteurs, d'un système sociospatial symbolique contextualisé (Champollion, 2007). Cette approche fonde le concept d'intelligence territoriale, qui rend compte des processus cognitifs que les communautés élaborent pour assurer le développement équitable de leur territoire (Girardot, 2004). Le concept suppose une méthode collaborative et vise un double objectif de développement: d'une part, avec la mise en place d'actions conceptuelles nécessaires aux transformations des consciences (Bozzano, 2008); d'autre part, en visant l'interrogation des pratiques dans des espaces déterminés où de nouvelles postures professionnelles sont susceptibles d'émerger. Le processus de territorialisation participe à l'intelligibilité des processus éducatifs; il caractérise le lien entre un territoire et le système de valeurs, les perceptions, les points de vue des acteurs, leurs connaissances, les stratégies citoyennes ou une forme de pouvoir d'agir qui transforme en permanence un espace de vie, à la recherche d'un équilibre.

\section{Force, faiblesse et complexité du développement durable}

$5 \quad$ En 1987, le développement durable émerge avec le rapport Brundtland ${ }^{4}$, se distinguant de la notion d'environnement, mais le DD est encore un concept en construction. Eu égard au vaste champ qu'il recouvre, il n'a pas, du moins chez les enseignants, de représentation claire de l'ensemble des dimensions qui le caractérisent. Ce n'est pas non plus une notion, dans le sens où il renvoie à des savoirs flous en cours d'élaboration (Alpe, 2006); et malgré une prise en compte internationale, ce point de vue sur le développement n'est en aucun cas universel. Il existe de nombreuses conceptions du développement, fondées sur d'autres rapports entre l'homme et son milieu de vie, inspirées par "une cosmogonie différente de la nôtre " (Sauvé, 2007). Pour certains, la notion de durabilité justifie des pratiques de développement qui creusent l'écart entre riches et pauvres, poursuivant l'occidentalisation du monde (Latouche, 2004). La pauvreté, contre laquelle veut lutter le développement durable, est perçue comme un problème et non pas comme un résultat du mal développement (Sauvé, 2007). Sa force, en revanche, est d'être un outil d'interrogation de la complexité du monde, visant des prises de décision, selon une logique qui met à mal une construction figée du monde. Dans une dialectique incessante entre la partie et le tout, les actions locales doivent être pensées en cohérence avec le système planétaire, et inversement une vision d'ensemble ne peut ignorer le travail local. La notion de durabilité se rapporte à une échelle de temps long qui perturbe nos repères pour penser les résultats d'une action si lointains que ses contemporains n'en connaîtront pas les effets. Cette nouvelle vision spatio-temporelle du monde vivant révèle une responsabilité collective relative à un mode de "vivre ensemble » qui engage l'avenir au-delà du temps vécu de chacun. Il s'agit donc de penser une citoyenneté hors de notre horizon quotidien, selon une 
temporalité qui conditionne "[...] la construction et (de) l'expression du sens" (Audigier, 2011, p. 39). À n'y pas prendre garde, le DD risque de se réduire à du développement économique durable et l'éducation devenir l'outil correspondant au service d'un programme «politico-économique mondial de développement durable " (Sauvé, 2007).

6 Par définition, le développement durable articule trois domaines : celui de l'écologie et du respect de l'environnement, celui des rapports sociaux avec l'équité sociale et la solidarité entre les sociétés, et celui de l'économie viable, qui considère que les ressources ne sont pas inépuisables. Il en découle que, sur un plan éducatif, le DD fait émerger trois dimensions : psychologique cognitive, due à l'élargissement du registre spatio-temporel qui tend vers un inconnu et rend nécessaire une autre façon de penser le temps et l'espace; politique, avec l'émergence d'une nouvelle citoyenneté, qui vise un autre rapport au mode de vie collectif dans une société élargie, au-delà du rapport au vivant (les ressources minérales font partie du problème) et du connaissable ; enfin épistémologique, avec la construction d'un nouveau champ de savoirs pluridisciplinaires lié à une culture donnée. En révélant la complexité du monde, le DD crée de nouvelles interactions disciplinaires. Le croisement et l'articulation des domaines et des dimensions projettent un ensemble complexe de problématiques éducatives qui soulignent le sens politique des savoirs ancrés dans le tissu social (Vivien, 2005). Les connaissances doivent élucider les divers modes d'équilibre homme/milieu de vie/environnement planétaire - et éclairer les nouveaux rapports entre les disciplines. Elles induisent par ailleurs des renversements de postures enseignantes qui ne sont pas des allants-de-soi. Une enquête menée auprès d'enseignants de collège et lycées (Boyer et Pommier, 2005) fait ressortir qu'en matière d'éducation au développement durable, tout semble devoir être transformé chez les enseignants : des situations éducatives à leur rôle et à leur posture.

\section{La méthode : une perspective collective, quantitative et explicative}

\section{Conditions de l'expérience}

7 Le travail d'investigation repose sur deux enquêtes conduites à un an d'intervalle auprès de populations d'enseignants à Marseille et en Italie, dans le Piémont et le Val d'Aoste $^{5}$, en utilisant la méthode des questionnaires. La première enquête avait fait ressortir chez les enseignants marseillais et valdôtains des caractéristiques distinctives entre développement durable et environnement (Floro, 2011). Ce premier travail a permis de construire un second questionnaire, centré sur le développement durable et adressé à des enseignants. Ce sont les résultats de ce second questionnaire, administré dans les trois territoires, Marseille, Val d'Aoste, Turin Piémont, que nous présentons dans ce texte. Les populations enquêtées sont les suivantes : en France, 19 enseignants d'un collège des quartiers sud de Marseille, 10 femmes et 9 hommes, issus de toutes les disciplines ont répondu. La passation s'est appuyée sur une structure collective associative : l'Observatoire des Quartiers Sud de Marseille ${ }^{6}$. Un enseignant du collège, membre de cet observatoire, a suivi et relancé ses collègues. Dans le Piémont italien, 83 enseignants de tous niveaux, 10 hommes et 73 femmes, issus des zones urbaines de montagne (Turin) et rurales de montagne (Oulx et Susa) ont répondu. La passation s'est 
faite par l'intermédiaire d'une équipe de l'USCOT, de l'Université de Turin, qui a joué le rôle de relais. Pour le Val d'Aoste, 33 enseignants de tous niveaux, 26 femmes et 7 hommes ont répondu. La passation s'est faite à Aoste, au cours d'une formation qui réunissait les enseignants de la vallée dans un but de titularisation. En tout, 135 enseignants ont été interrogés, dont huit seulement avaient moins de deux années d'expérience.

8 Le questionnaire a visé le recueil d'information le plus objectivement possible, afin de saisir des caractéristiques singulières d'une population, mettre en évidence des faits psychologiques et, ou sociaux et les facteurs qui les déterminent. Il présente 15 catégories de sens centrées sur le développement durable. La première question de ce nouveau questionnaire avait pour objectif de mobiliser les savoirs sur le DD, et demandait de préciser cinq points essentiels parmi les 15 proposés. Elle était suivie par sept autres questions visant à approfondir: valeurs, savoirs évoqués, perspectives d'action éducatives, actions entreprises dans et hors l'école. Enfin, une dernière question tentait de repérer si la catastrophe nucléaire de Fukushima du 11 mars 2011 avait induit une évolution des problématiques.

\section{Le facteur territorial}

9 La complexité du territoire induit un travail continuel d'analyse allant de la surface à la profondeur, du macroscopique au microscopique, du visible au caché. Les éléments caractéristiques présentés ci-dessous ne sont pas exhaustifs ; ils permettent seulement de porter un autre regard sur les similitudes et les spécificités des réponses obtenues. $\mathrm{Si}$ une part des questions posées vise la posture des professeurs relatifs à leur engagement dans les domaines de l'éducation au développement durable, les facteurs choisis caractérisent leur position par rapport aux lieux et aux politiques qui développent un patrimoine local en lien avec des problématiques énergétiques et environnementales. À Marseille, l'orientation de l'action du Parc national des Calanques vise la protection du milieu naturel avec un programme d'aménagement et de préservation de l'habitat et de la biodiversité. Ses problèmes majeurs sont les incendies et l'accueil des visiteurs, nombreux à cause de la proximité de la ville. Les actions à vocation pédagogique, à destination des différents publics, visent l'amélioration de la qualité des eaux marines, des espaces terrestres et encouragent des « éco-gestes " ${ }^{7}$. Les parcs Italiens du Piémont privilégient leur patrimoine historique et culturel associé à l'économique. Le parc Stupinigi, à $10 \mathrm{~km}$ de Turin et constitué de zones boisées et agricoles, a une action autant environnementale, culturelle, historique ou artistique ${ }^{8}$, qu'économique. Le Gran Bosco Salbertrand à $60 \mathrm{~km}$ de Turin, associe la découverte de la nature, l'histoire et le tourisme. C'est une aire d'altitude et de protection d'une faune et d'une flore de montagne, aménagée pour la randonnée. Il propose la découverte de sentiers historiques, comme celui emprunté par les armées de Charlemagne pour passer en Italie. En Val d'Aoste, le parc du Grand Paradis privilégie l'action écologique. Succédant à une réserve royale ${ }^{9}$, il est devenu parc national en 1922 et s'est engagé dans diverses actions pour limiter ou éliminer l'utilisation de produits toxiques dangereux pour l'environnement, promouvoir les énergies propres et les technologies éco-compatibles. Quant aux politiques énergétiques et environnementales, le Val d'Aoste exerce une gouvernance régionale autonome. Son relief montagneux et ses ressources naturelles en eau lui permettent une production autosuffisante d'énergie hydroélectrique. L'assessorat régional "aux activités productives" vise la promotion des sources 
d'énergie propres et renouvelables et le développement de technologies innovatrices. La Compagnie valdôtaine des eaux gère la structure des centrales dispersées sur le territoire; l'électricité est ensuite distribuée par une entreprise à participation régionale ${ }^{10}$. En termes de logique d'action citoyenne collective et cohérente, la population du Val d'Aoste a su refuser par referendum l'implantation d'un incinérateur à une écrasante majorité de $94 \%$ des voix. Pour la région Piémont en revanche, un ensemble de projets de communication secoue la région : d'une part, celui de la liaison électrique avec la Savoie ; d'autre part, celui du Train à Grande Vitesse (TGV/TAV) liant Turin à Lyon. Le premier a été soumis à une enquête publique, et des inquiétudes ont été exprimées, relatives au coût croissant de l'électricité et aux risques liés à sa production. Le second mobilise les citoyens. Des comités de soutien « No TAV» naissent de part et d'autre de la frontière.

\section{Les stratégies nationales de développement durable (SNDD) et leur impact sur l'éducation}

10 Les politiques de développement durable en France et en Italie sont comparables. Depuis le Sommet de la Terre de Rio en 1992, les deux pays ont pris des engagements internationaux similaires, réaffirmés par la suite dans une stratégie européenne de développement durable ${ }^{11}$, pour lutter contre le changement climatique en réduisant les émissions de gaz carbonique. Ces stratégies visent des objectifs et des actions permettant d'atteindre un équilibre entre les aspects environnementaux, sociaux, culturels et économiques d'un territoire.

11 En Italie, quatre principes fondent la Stratégie nationale d'action ${ }^{12}$ : le changement climatique et la couche d'ozone, la protection et la valorisation à long terme de la nature et de la biodiversité, la qualité de l'environnement et de la vie dans les aires urbaines, l'exploitation des ressources et le traitement des déchets. Elle vise la cohérence des politiques environnementales sous la responsabilité du Ministère de l'Environnement et de tous les partenaires concernés, notamment des collectivités locales. Dans tous les secteurs, les actions se font au niveau local (régions, provinces, communes); contrairement à la France, la politique environnementale n'est pas soumise aux décisions du gouvernement central. De ce fait, il n'y a pas d'actions réelles de développement durable coordonnées au niveau national.

Le gouvernement français a reconnu l'engagement international de l'État dans le domaine du Développement durable par l'approbation du 3 juin 2003 qui se fonde sur six grands axes stratégiques: les citoyens acteurs du développement durable; l'information et l'éducation; les territoires et les environnements urbain et rural; les activités économiques incluant producteurs et consommateurs énergie, transports et agriculture; la prévention des risques, la pollution et autres problèmes environnementaux et sanitaires; le rôle du gouvernement. La Stratégie nationale du développement durable encadre les actions du gouvernement dans le champ du développement durable et plus généralement dans toutes les politiques publiques. Elle a un aspect transversal dans le sens où, au niveau national, chaque ministère est responsable de la mise en œuvre et du suivi des actions de son domaine d'activités. La mise en œuvre éducative repose sur des enseignements obligatoires inscrits dans le socle commun de connaissances et de compétences ${ }^{13}$. L'orientation actuelle en France va dans le sens d'une transition énergétique écologique. 

italien n'est pas soumis à des programmes de développement durable. Pas de prescription, mais la «Charte de Fiuggi $»^{14}$ de 1997, qui fixe les principes et les indications générales d'une "éducation à l'environnement vers un développement durable dans les écoles ». Ces principes associent, dans une éducation citoyenne, une méthode participative et une éducation globale à l'environnement, souvent développée par les enseignants de sciences ou de géographie. Chaque école, étant autonome, développe des projets et fait appel à des spécialistes extérieurs. Ces éléments locaux entrent souvent en conflit avec la Stratégie nationale de développement durable.

\section{Les résultats}

\section{Caractérisation générale du développement durable par les enseignants}

Cette seconde enquête montre que pour ces enseignants, aujourd'hui, le DD renvoie à des pratiques de consommation d'énergie, de protection du milieu de vie, mais en aucun cas à une utopie. Les définitions qu'ils attribuent au développement durable (Tableau 1) font ressortir un ensemble de quatre catégories :

$1^{\text {ère }}$ catégorie. Chaque caractéristique est évoquée par les sujets avec une fréquence supérieure à $50 \%$. Elle renvoie aux actions de gestion des ressources et de protection de la nature, qui caractérisaient l'environnement précédemment. Ces réponses sont sans doute liées à l'émergence de la notion de danger qui apparaissait peu dans la première enquête (Floro, 2011).

$2^{\mathrm{e}}$ catégorie. Les fréquences des réponses oscillent entre 40 et $50 \%$. Les réponses sont de nature conceptuelle : les valeurs, l'éducation, la conscience d'un autre rapport au monde. Par rapport à leur absence dans la première enquête, elles représentent des indicateurs d'évolution.

$173^{e}$ catégorie. Elle regroupe des réponses dont les fréquences varient entre 25 et $40 \%$, qui marquent l'instabilité de certaines caractéristiques, avec la montée du sentiment de danger, de la gouvernance, la volonté de gérer les ressources et de protéger la nature, et l'importance de la biodiversité et du vivant. D'un autre côté, les réponses centrées sur le tri sélectif, et les questions d'environnement, et d'énergie restent au même niveau que dans l'enquête précédente.

$184^{\mathrm{e}}$ catégorie. De nature hétérogène, avec des fréquences inférieures à $20 \%$, elle montre que les caractéristiques liées au temps frémissent faiblement. Par ailleurs, il est surprenant de constater que l'écologie, légitimement attendue, est peu citée (17\%).

\section{Répartition globale des réponses en fonction de chaque territoire}

Le tableau 2 présente la répartition des réponses en fonction de chaque territoire. Il fait apparaître, qu'excepté la gestion des ressources qui domine avec celle de protection de la nature et celle de danger qui émerge, il existe des disparités entre territoires. Les enseignants marseillais s'orientent vers des actions de protection quotidiennes de proximité par le tri sélectif; le Val d'Aoste se démarque par une évocation des dispositifs d'éducation et de formation; pour le Piémont les réponses sont plus notionnelles, il 
s'agit de valeurs et de protection de la nature ; il est à signaler toutefois que pour la partie urbaine, la protection de la nature prédomine.

Tableau 1 : Catégorisation selon les fréquences des caractéristiques attribuées au DD, pour l'ensemble des territoires étudiés.

\begin{tabular}{|c|c|c|c|c|c|c|c|}
\hline \multicolumn{2}{|l|}{ Actions } & \multicolumn{2}{|c|}{ Domaine conceptuel } & \multicolumn{2}{|c|}{ Instabilité, variations } & \multicolumn{2}{|l|}{ Hétérogènéité } \\
\hline Gestion des ressources & $68 \%$ & Valeurs & $48 \%$ & Danger & $38 \%$ & Écologie & $17 \%$ \\
\hline Protection nature & $56 \%$ & Éducation & $46 \%$ & Tri & $38 \%$ & Temps & $17 \%$ \\
\hline & & Conscience au monde & $45 \%$ & Biodiversité & $36 \%$ & Éléments chimiques & $9 \%$ \\
\hline & & & & Gouvernance & $32 \%$ & Utopie & $0,7 \%$ \\
\hline & & & & Environnement & $26 \%$ & & \\
\hline & & & & Énergie & $25 \%$ & & \\
\hline
\end{tabular}

Tableau 2 : Fréquences des caractéristiques affectées au DD pour chaque territoire

\begin{tabular}{|r|c|c|c|c|}
\hline & Marseille & Piémont Urbain & Piémont Rural & Val d'Aoste \\
\hline Gestion Ressources & $\mathbf{6 8} \%$ & $\mathbf{6 2} \%$ & $\mathbf{7 0} \%$ & $\mathbf{7 2} \%$ \\
\hline Protection nature & $52 \%$ & $\mathbf{6 5} \%$ & $57 \%$ & $45 \%$ \\
\hline Valeurs & $42 \%$ & $58 \%$ & $50 \%$ & $36 \%$ \\
\hline Éducation & $31 \%$ & $48 \%$ & $40 \%$ & $60 \%$ \\
\hline $\begin{array}{r}\text { Conscience d'un autre } \\
\text { rapport au monde }\end{array}$ & $52 \%$ & $37 \%$ & $57 \%$ & $36 \%$ \\
\hline Danger & $36 \%$ & $39 \%$ & $40 \%$ & $36 \%$ \\
\hline Tri & $\mathbf{6 8} \%$ & $37 \%$ & $25 \%$ & $39 \%$ \\
\hline Bio Diversité & $31 \%$ & $27 \%$ & $45 \%$ & $42 \%$ \\
\hline Gouvernance & $31 \%$ & $18 \%$ & $40 \%$ & $42 \%$ \\
\hline Environnement & $36 \%$ & $30 \%$ & $10 \%$ & $24 \%$ \\
\hline Énergie & $36 \%$ & $23 \%$ & $10 \%$ & $33 \%$ \\
\hline École & $21 \%$ & $23 \%$ & $17 \%$ & $6 \%$ \\
\hline Temps & 26 & $23 \%$ & $15 \%$ & $9 \%$ \\
\hline Utopie & $5 \%$ & $11 \%$ & $5 \%$ & $9 \%$ \\
\hline & & $2 \%$ & $0 \%$ & $0 \%$ \\
\hline
\end{tabular}

\section{La montée du sentiment de danger}

Nous avons ensuite interrogé la montée observée du sentiment de danger. A-t-elle un lien avec la catastrophe nucléaire de Fukushima? Les résultats montrent que $53 \%$ des enquêtés disent ne pas avoir changé de point de vue, seulement $20 \%$ répondent par l'affirmative. En revanche, $27 \%$ ne donnent pas de réponses. Les changements portent sur la prise de conscience des diverses formes de danger nucléaire, les difficultés à maîtriser cette technologie et les déchets qui en résultent (30\%). Il faut la remplacer par d'autres formes plus sûres de production énergétique. Les questions inhérentes au DD sont liées à une volonté politique (26\%) qui n'est pas toujours transparente (15\%). Pour ceux qui n'ont rien changé à leur conception, $47 \%$ des réponses n'ont pas d'explication 
et ces événements confirment le nécessaire refus de l'énergie nucléaire et une responsabilité attribuée au politique. En revanche, la responsabilité politique du Japon n'est quasiment pas citée.

21 Existe-t-il des effets de territoire sur ces évolutions? On observe en effet certaines disparités. Pour le Val d'Aoste, maîtriser le nucléaire n'est jamais évoqué. Pour le Piémont, c'est une question pour $25 \%$ de la population, et la responsabilité politique de la région est engagée (37\%), en particulier dans les projets conflictuels sur l'énergie et les transports. En revanche, à Marseille, la question du nucléaire est vive pour $57 \%$ des enseignants et s'inscrit dans une problématique globale pour $85 \%$ d'entre eux - qui semblent évacuer la responsabilité politique. Alors que $53 \%$ n'ont rien changé à leur conception, $47 \%$ des répondants n'ont pas d'explication. Plusieurs (19\%) étaient déjà persuadés que la question de l'énergie nucléaire était à remplacer. Mais l'importance des réponses sans explications, entre $35 \%$ et $50 \%$, laisse penser qu'elles sont plus de nature idéologique que fondées sur un savoir scientifique ou une rationalité. Ensuite, l'importance des non réponses pourraient représenter une forme inavouée d'acceptation de ce type d'énergie. Les événements japonais ne produisent pas de changement de point de vue sur des valeurs associées au développement d'une nouvelle citoyenneté. Aucune réponse ne tente de faire le point sur ce qui aurait pu changer dans une perspective d'intelligence territoriale planétaire, impliquant une gouvernance mondiale qui regrouperait l'ensemble de l'humanité face à l'étendue du problème. S'il existe une vision commune sur la question de la source énergétique du futur, l'analyse fait apparaître un effet de territoire. Les enseignants français confrontés au problème de l'énergie nucléaire font de sa maîtrise un enjeu majeur; en revanche, dans le Val d'Aoste, autonome sur le plan de l'énergie, on ignore ce redoutable écueil. Dans le Piémont, confronté au double problème du train et du transport d'électricité, les enseignants portent un regard critique sur le champ politique.

\section{L'ancrage de l'action éducative dans et hors de l'école et les savoirs en jeu}

Sur le plan des savoirs, le flou persistant confirme les difficultés d'une distinction entre éducation à l'environnement, et éducation au développement durable. La transversalité représente pour plus de $30 \%$ des enseignants une façon de penser le développement durable, "on le retrouve partout, dans toutes les disciplines». Ce type de posture relatif aux disciplines renforce l'idée d'une difficulté à problématiser le champ, qui permettrait une conception non seulement transversale, mais interdisciplinaire. Les disciplines majoritairement citées sont celles des enseignants. Nous les avons classées en sciences humaines, sciences de la vie et de la terre, éducation à l'environnement, citoyenneté, étude du temps. Ceux qui proposent un champ disciplinaire évoquent, pour $20 \%$ d'entre eux, une discipline unique et $15 \%$ énumèrent surtout celles qu'ils enseignent. Enfin, $11 \%$ parlent de sciences en général. De façon générale, le développement durable n'est jamais perçu comme un élément producteur de problématiques ou comme outil d'interrogation du monde. Les questions relatives aux actions éducatives distinguant les lieux dans et hors de l'école posent problème aux enseignants. Presque $25 \%$ des professeurs ne répondent pas pour les actions dans l'école et $30 \%$ pour les actions en dehors. Dans l'école, les activités évoquées dessinent cinq catégories : le tri sélectif, les éducations à (l'environnement, la santé...), les actions sur l'extérieur de l'école, les actions visant les valeurs, les enseignements scolaires. Pour les actions éducatives hors du cadre scolaire, les activités citées fondent une 
catégorisation associée à la précédente : le tri, la formation, l'information, le débat, les actions de la vie domestique, les valeurs et les postures éthiques, l'engagement social hors de son lieu d'habitation. Si certaines actions sont cohérentes dans et hors du cadre scolaire, des disparités apparaissent pour chaque territoire en fonction des thèmes et de ces mêmes espaces. À Marseille, les actions de DD sont tournées vers le milieu proche, aussi bien dans l'école (27\%) qu'à l'extérieur ( $22 \%)$, et sur des disciplines scolaires. Le tri n'est pas un contenu éducatif prioritaire, mais hors école c'est une action du quotidien (22\%). Pour le Val d'Aoste, de la même façon que pour Marseille, les actions scolaires (15\%), comme les actions hors école (17\%), sont centrées sur le milieu proche. En revanche, le tri est un support éducatif dans le cadre scolaire (21\%) et hors scolaire ( $25 \%)$. C'est là que l'engagement social est le plus fort (15\%). Pour le Piémont, qui s'engage le plus, $88 \%$ de réponses, les actions éducatives sont des éducations à... (18\%), qui semblent en cohérence avec des actions quotidiennes centrées sur le milieu proche (19\%). C'est en Piémont que les actions éducatives scolaires sont le plus tournées vers l'extérieur de l'école ( $16 \%$ ) et vers un univers quotidien social, local (12\%).

\section{Discussion sur les difficultés de perception de l'objet}

Les points caractéristiques du développement durable se rapportent à deux grands types d'actions : la gestion des ressources et la protection de la nature. L'ensemble des réponses fait apparaitre une absence de référence au temps et en particulier au « temps long (Braudel, 1958), qui caractérise la problématique durable. La notion percute vraisemblablement une façon majoritaire de penser un temps présent, telle «une dictature de l'instant» (Audigier, 2011), et témoigne de la difficulté au plan cognitif d'intégrer une pluralité d'échelles de temps (Rosa, 2010) et d'espace. Les enseignants interrogés décrivent peu d'actions précises et ne parlent pas des conditions de mise en œuvre de leurs propositions d'action. Ils sont pris dans des tensions liées au débat sur le développement durable. D'un côté, ils participent comme citoyens à la production de ces interrogations, de l'autre ils sont chargés de transmettre des contenus opaques. Cependant, ces savoirs fragiles liés aux questions vives ont la force des questions fondamentales posées à la société humaine et, par là, à chacun. Les " normes éthiques » sont «[...] au cœur des prises de position, des choix et des actions des acteurs individuels et collectifs » (Audigier, 2011, p. 40).

La disparité des réponses en fonction du territoire, témoigne d'effets locaux. Le développement durable est expliqué par un environnement décrit en termes d'investissement humain d'un espace spécifique local, et l'engagement dans l'action quotidienne vise clairement la protection de l'environnement proche. Un ensemble de facteurs peut expliquer ces résultats : l'absence ou la présence de programmes et de contenus disciplinaires prescripteurs, le degré d'autonomie des établissements scolaires, les enjeux des politiques énergétiques, les projets d'aménagement des territoires et des espaces protégés, voilà autant d'événements susceptibles de développer un pouvoir d'agir, envisagé comme un rapport de forces conflictuel, qui n'est pas de la violence, mais permet « une action sur les actions possibles » (Deleuze, 1986). Ces événements ${ }^{16}$, induisent des problématiques d'espace et de temps, qui affectent des points de vue figés sur la gouvernance. Face à un danger planétaire, les frontières politiques n'existent plus, la nation disparaît au profit de la solidarité 
internationale, car rien n'arrête les nuages radioactifs ou les nappes d'hydrocarbure. Le savoir prend un sens universel, la connaissance des territoires (zone sismique, inondable...) rend nécessaire une prise de décision globale collective, une gouvernance mondiale démocratique où la planète remplace la cité. Penser une citoyenneté planétaire peine à entrer dans les programmes éducatifs et dans les esprits. Mais les savoirs que sous-tend le DD rendent incontournables une solidarité internationale, et militent contre des enjeux uniquement économico-financiers.

\section{Conclusion : territoire et déterritorialisation, appuis et obstacles}

Les citoyens-enseignants semblent avoir des difficultés à gérer simultanément deux types de pensée : celle du local et celle du global, celle de la partie et celle du tout. L'EDD demande de penser deux choses simultanément, tout en faisant le pari que l'homme se transformera lui-même. L'humanité aurait un grand intérêt à s'inscrire dans un nouveau contrat naturel, comme une forme de savoir sur la nature, dans une logique de Sujet à Sujet (Serres, 1987). Ce qui suppose une double transformation: d'abord sur le plan psychologique pour repenser un monde complexe à la fois physique, comme espace et comme temps ; mais aussi humain et vivant, ensuite, sur le plan du rapport à la planète, qui suppose d'établir avec elle un lien qui dépasse celui de protecteur, Homme, à protégée, Planète, évacuant l'idée de rapport dominant-dominé. La nature est-elle un musée ? Il s'agit de penser une situation d'échange équitable avec le vivant et le non-vivant, pris comme ressource matérielle épuisable, soumise à un mode d'extraction qui lui est lié, comme effet produit par son utilisation, mais aussi comme résidu à traiter après son utilisation. Ce passage à l'éducation au développement durable s'explique à travers la relation que le sujet enseignant et citoyen construit avec l'objet flou développement durable. Mais le développement durable est-il un objet d'éducation? Sa nature en fait une question vive et la façon dont les acteurs l'évoquent s'appuie en grande partie sur des réalités sociales locales. Cette posture décrit les caractéristiques d'une éducation aux environnements, qui ont un rôle d'appui. Ils permettent en effet de donner du sens à des actions citoyennes et de développer une perspective éducative active, par des postures de défense et de protection du milieu (faune, flore, patrimoine, ressources, etc.). Mais cette centration sur le territoire est un obstacle dans le sens où il occulte les transformations de temps, d'espace, de gouvernance, qui conditionnent une nouvelle façon de penser les rapports au monde. Mais poser le problème dans un cadre territorial permet de penser le passage de l'environnement à celui de développement durable comme un processus de déterritorialisation (Deleuze et Guattari, 1980). La déterritorialisation décrit le processus d'extraction d'un individu ou d'un groupe inscrit dans un ensemble de relations qui organisent leur territoire. Décontextualiser ces relations est nécessaire à leur actualisation dans d'autres contextes, comme processus de libération d'une pensée engluée dans des problématiques uniquement locales. Les auteurs distinguent "déterritorialisation relative et absolue» (Deleuze et Guattari, 1980). La déterritorialisation relative laisse la place à une reterritorialisation, ce que ne suppose pas la déterritorialisation absolue. Ce concept permet de comprendre la nécessité de s'extraire du poids des faits locaux. Ces faits sont inducteurs de problématiques certes, mais réducteurs. Car ils empêchent de prendre de la distance par rapport à un univers 
quotidien restreint, pour parvenir à une vision complexe de cet ensemble de questions et pour mieux s'y réinscrire. Le territoire médiateur pourrait agir aussi comme un attracteur qui réduit le champ de vision à un milieu de vie proche.

Mais il ne s'agit pas non plus de nier le local et de dériver vers un modèle occidental de développement comme archétype universel, qui viserait une civilisation unique, dénaturée, sans dynamisme et fragile. Si le territoire est un point d'appui, qui donne du sens aux savoirs, une réalité à une discipline, une dimension émotionnelle à l'acte d'apprendre, s'il permet une prise de conscience progressive des réalités du milieu de vie et oriente une action pensée collectivement, en rapport avec la protection de l'environnement et le développement équilibré, il peut aussi devenir un obstacle, empêchant la prise de hauteur et l'ouverture à un ailleurs. Le processus éducatif fait apparaître un lien potentiel entre l'enseignant-citoyen et le développement durable médiatisé par le territoire. C'est en partie de la gestion de ce territoire médiateur que dépendra l'apprentissage infini du monde, comme processus de développement humain singulier.

\section{BIBLIOGRAPHIE}

Alpe, Y. (2006). Existe-t-il un déficit culturel chez les élèves ruraux ? Revue française de pédagogie, juillet-août-septembre, 156, 75-88.

Audigier, F. (2011). Penser les temporalités pour penser les questions sociales vives. Éduquer au développement durable pour construire l'avenir. Dans Legardez, A. et Simonneaux, L. (dir.). Développement durable et autres questions d'actualité. Questions socialement vives dans l'enseignement et la formation (p. 33-52). Toulouse : Educagri.

Audigier, F. (2008). Forme scolaire forme sociale. Babylonia 3/08.

Boyer, R. et Pommier, M. (2005). La généralisation de l'éducation à l'environnement et au développement durable vue par les enseignants du secondaire. Lyon : INRP.

Bozano, H. (2008). Compréhension et développement d'un territoire, un nouveau réseau.

Communication présentée à la $6^{\mathrm{e}}$ Conférence internationale d'intelligence territoriale. Besançon : Université de Franche-Comté.

Bozonet, J.-P. (1992). Des monts et des mythes : l'imaginaire social de la montagne. Grenoble : Presses Universitaires de Grenoble.

Braudel, F. (1958). La longue durée. Annales E.S.C., octobre-novembre, 4.

Champollion, P. (2008). La territorialisation de l'école au regard de l'égalité des chances : frein ou levier? Éléments de cadrage et de réflexion développés principalement à partir des territoires ruraux isolés et montagnards. Symposium « territoire » du colloque ACSE « efficacité et équité en éducation ». Rennes, novembre 2008.

Deleuze, G. (1986). Foucault. Paris : Éditions de Minuit.

Deleuze, G. et Guattari F. (1980). Mille plateaux. Paris : Éditions de Minuit. 
Floro, M. (2011). Développement durable et questions socialement vives, une approche territorialisée du discours enseignant. Dans Legardez, A. et Simonneaux, L. (dir.). Développement durable et autres questions d'actualité. Questions socialement vives dans l'enseignement et la formation (p. 163-179). Toulouse : Educagri.

Girardot, J.-J. (2004). Intelligence territoriale et participation. ISDM, 16, art. 161.

Grelet, Y. (2006). Des territoires qui façonnent les parcours scolaires des jeunes, Publication $d u$ CEREQ, 288.

Gumuchian, H. (2008). Un objet géographique pertinent en matière d'éducation. Diversité, ville école intégration, 155, 41-42.

Latouche, S. (2004). Survivre au développement. Collection Les Petits Livres, 55. Paris : Mille et une nuits.

Le Berre, M. (1992). Territoires. Dans Bailly et coll., Encyclopédie de géographie (p.617-638). Paris : Economica.

Legardez, A. (2007). Éduquer au développement durable et/ou faciliter la co-construction de savoirs critiques sur l'éco-citoyenneté. Communication au Symposium REF « Dimensions individuelles et collectives de l'éducation au développement durable : regards croisés et perspectives critiques », 9 et 10 octobre 2007, Sherbrooke.

Legardez, A. et Simonneaux, L. (2006). L'école à l'épreuve de l'actualité: Enseigner les questions vives. Paris : ESF Éditeur.

Legardez, A. et Simonneaux, L. (2011). Didactique des questions socialement vives. Répondre aux besoins de formation dans la société post-moderne. Dans Legardez, A. et Simonneaux, L. (dir.). Développement durable et autres questions d'actualité. Questions socialement vives dans l'enseignement et la formation (p. 163-179). Toulouse : Éducagri.

Ravetz, J.R. (1997). Simple scientific truths and uncertain policy realities. Studies in science education, 30,1 .

Rosa, H. (2010). L'accélération. Paris : La Découverte.

Sauvé, L. (2007). L'équivoque du développement durable. Chemin de Traverse, 4, 31-47. Serres, M. (1987). Le contrat naturel. Paris : Éditions Françoise Bourin.

Vincent, G. (2008). La socialisation démocratique contre la forme scolaire. Éducation et francophonie, volume XXXVI (2).

Vivien, F.-D. (2005). Le développement soutenable. Collection Repères. Paris : La découverte.

\section{NOTES}

1. ANR/ED2AO Éducation au Développement Durable, Appuis et Obstacles, 2009 et 2013, équipe de l'IUFM AMU d'Aix-en-Provence laboratoire ADEF sous la direction d'Alain Legardez, et le pilotage national de Jean-Marc Lange, Université de Rouen.

2. 3D : Démarche de Développement Durable.

3. PNS : Post Normal Science, concept que l'on doit à Funtowitcz S.O., Ravetz, J.R., 1993. Science for the post normal age, Futures, 25 (7)

4. Rapport intitulé : "Notre avenir à tous", il évoque un "développement qui répond aux besoins du présent sans compromettre la capacité des générations futures à répondre aux leurs ». 5. Régions italiennes de montagne. Le Val d'Aoste est autonome grâce au bilinguisme. 
6. Association loi de 1901, créée en juin 2008.

7. Il s'agit de la gestion des rejets divers, des partenariats avec les professionnels de la pêche et les associations d'usagers, du balisage et de l'aménagement des espaces.

8. Musée d'Art et d'Ameublement.

9. Créée par Victor-Emmanuel II en 1856 pour protéger le bouquetin. Il adhère aujourd'hui au protocole d'intégration pour la promotion du patrimoine public écologique.

10. Entreprise à participation régionale, Vallénergie.

11. Göteborg en juin 2001, 2002 au Sommet de Johannesburg (ONU).

12. Ministero dell'Ambiente e della Tutela del Territorio - (Stratégie d'action environnementale vers le développement durable en Italie). "Strategia d'azione ambientale per lo sviluppo sostenibile in Italia" - Approuvé par la CIPE le 2 août 2002. Délibération n 57. Publiée au journal officiel $n^{\circ} 255$ du 30 octobre 2002, ordre $n^{\circ} 205$.

13. Connaissance de l'impact sur l'environnement de nos activités techniques; référence à une attitude de responsabilité face à l'environnement, au monde vivant, à la santé; mention du développement durable comme un moyen de comprendre l'unité et la complexité du monde. BO $\mathrm{n}^{\circ} 14$ du 5 avril 2007.

14. Charte pour les principes d'éducation à l'environnement vers un développement durable, Séminaire National : a Scuola d'Ambiente, Fiuggi 24 avril 1997.

\section{RÉSUMÉS}

Ce texte présente la façon dont des enseignants de tous niveaux se positionnent face à la question du développement durable. Le problème posé dans le contexte des questions socialement vives est analysé dans la perspective d'une approche territorialisée. Les représentations que construisent les différents acteurs s'inscrivent dans un triptyque « représentations - pratiques - action", dont le territoire est une clé de lecture. Les résultats montrent que les caractéristiques du territoire influencent la façon d'aborder le développement durable et orientent les choix d'action éducative; deuxièmement, on observe qu'il est difficile pour les enseignants de penser de façon distincte développement durable et environnement.

This papers presents the way teachers at various levels position themselves with regard to sustainable development. The problem, addressed within the context of socially acute questions, is analyzed from the perspective of territory. Representations that different social actors construct are embedded in the threefold "representations - practices - action" for which territory is an interpretation key. Results show that the characteristics of territory influence the ways of apprehending sustainable development and they orient educational choices. We also notice that is difficult for teachers to envision differences between sustainable development and environment 


\section{AUTEUR}

\section{MICHEL FLORO}

Michel Floro est enseignant chercheur, psychologue, membre du comité de développement durable de l'Écoles Supérieures du Professorat et de l'Éducation. Engagé dans le défi sur l'inclusion du plan vert de l'AMU. Participe à des recherches internationales sur les questions de professionnalité liées aux processus éducatifs au développement durable et aux obstacles à l'éducation, selon une approche territoriale. Aborde des questions vives de décrochage scolaire, de pauvreté, de violence scolaire et de harcèlement. Membre fondateur de l'observatoire des quartiers sud de Marseille. 